\title{
On Generically Dependent Entities
}

\author{
Antony Galton \\ College of Engineering, Mathematics, and Physical Sciences, University of Exeter, UK \\ E-mail: apgalton@ex.ac.uk
}

March 15, 2016

\begin{abstract}
An entity $x$ is said to be generically dependent on a type $F$ if $x$ cannot exist without at least one entity of type $F$ existing. In this paper several varieties of generic dependence are distinguished, differing in the nature of the relationship between an entity and the instances of a type on which it generically depends, and in the light of this criteria of identity for generically dependent entities are investigated. These considerations are then illustrated in detail in a series of three case studies, covering shapes, linguistic entities such as letters, words and sentences, and collectives. Each case study examines how far the entities involved have robust identity criteria, and to the extent that they do not it is questioned whether they can be regarded as bona fide examples of generic dependent entities. Finally, in the light of this, a number of possible accounts that may be given of the ontological status of such entities are considered.
\end{abstract}

KEYWORDS: ontological dependence, generic dependence, identity criteria, shape, linguistic entities, collectives

\section{Ontological Dependence}

There are many ways in which one entity may be said to be dependent on another - see for example the enumeration given in $[30, \S 8.2]$. Amongst this variety of kinds of dependence, ontological dependence has been singled out as particularly interesting for ontology. Informally, an entity $a$ is said to be ontologically dependent on an entity $b$ if $a$ cannot exist without $b$ also existing. An example that has often been given is that a particular smile is ontologically dependent on a particular face: specifically, if this particular face did not exist, then neither could that particular smile. Further examples are enumerated in $[5, \S 2.1]$ and $[19, \S 1]$. Note that the dependence here is specific: the existence of one particular is dependent on the existence of a specific other particular. Thus this particular smile could not have been borne by a different face - if the face is different, the smile is different, however qualitatively similar it may be. Smith [32] notes that this is not necessarily a one-sided relation, as shown by the mutual dependence of the north and south poles of a magnet: neither can exist without the other. Although the literature of applied ontology tends to favour the term specific dependence, in the philosophical literature this kind of dependence, of one particular on another, is generally referred to as rigid dependence.

Precisely how this kind of dependence should be characterised has been the subject of considerable debate amongst philosophers. In the "modal/existential" approach (so-called by [8]), the 'cannot' of the informal characterisation of dependence is interpreted by means of a modal operator, giving:

$$
a \text { is dependent on } b={ }_{\operatorname{def}} \square(\operatorname{Exists}(a) \rightarrow \operatorname{Exists}(b))
$$

or one of the more refined variants of this (designed to prevent entities being dependent on themselves, or dependent on arbitrary necessarily-existing entities such as numbers) discussed by Simons [30]. Definitions in this spirit feature in numerous discussions of ontological dependence, generally without any 
indication of the precise logic governing the modal operator; S5, used by [4], seems a likely enough candidate, but there has not to my knowledge been a systematic discussion of what difference alternative choices of modal logic would make to the modal/existential account of ontological dependence.

Fine [8] by-passes all this, suggesting that 'the necessary connection between the two existences is too loose to do justice to the facts of dependence'; instead, he proposes to 'substitute the notion of essence for that of necessity'. In Fine's account, ' $a$ cannot exist without $b$ existing' is interpreted to mean, not just that $b$ 's existence is a necessary precondition of $a$ 's existence, but that it is an essential property of $a$ that it exists only if $b$ does (see also $[5,18]$ ). Fine replaces the necessity symbol $\square$ in (SD) by the symbol $\square_{x}$, which is understood to mean 'it is true in virtue of the identity of $x$ that'; similar replacements could be made in the formulae below.

One reason for taking ontological dependence seriously is that it plays a key role in determining what the most fundamental constituents of an ontology are. This privileged role is accorded to those entities that are deemed to be ontologically independent, that is, their existence is not dependent on the existence of anything else; they exist, as it were, "in their own right". Traditionally, such entities have been called substances - the link between substances and ontological independence is explicitly drawn by, amongst others, Lowe [21], who cites Descartes in this connection. Thus, for example, one might argue as to whether objects or their properties are more fundamental: it is more conventional to suppose that objects are independent and their specific properties (for example, the redness and roundness of this ball) are dependent upon them; but many philosophers at different times have claimed that, on the contrary, the specific properties or tropes are what exist independently, and the objects, being "nothing but" bundles of tropes, are dependent upon them.

Specific, or rigid, dependence is a relationship between particular individuals, but it is very often possible to formulate general statements of dependence at the level of types rather than individual instances. Thus not only is this smile dependent on this face, but also smiles in general are dependent on faces in general. This is a case of generalised specific dependence, since what it says is that each individual smile is specifically dependent on some individual face. This can be expressed by a formula of the following type:

$$
F \text { s are dependent on } G \mathbf{s}={ }_{\text {def }} \forall x(F(x) \rightarrow \exists y(G(y) \wedge \square(\operatorname{Exists}(x) \rightarrow \text { Exists }(y))),
$$

where, as suggested above, the $\square$ can be replaced by $\square_{x}$ if Fine's essentialist interpretation is preferred.

Quite distinct from this generalised specific dependence, generic dependence is a dependency relation between individuals and types. Here a particular entity depends for its existence on the existence of another entity of a particular type, but not on any one specific entity of that type. This may be expressed by the formula

$$
a \text { is generically dependent on } G s={ }_{\text {def }} \square(\operatorname{Exists}(a) \rightarrow \exists x G(x)) .
$$

As an example, a human being depends for its existence on there being atoms that constitute its body; but it does not depend on any specific collection of atoms, as is evident from the fact that the same human being persists over many years despite a wholesale turnover of the atoms. The human is generically, but not specifically, dependent on collections of atoms. Similarly, a queue only exists because there are people queuing, so the queue is ontologically dependent on queuers: but it is clearly not dependent on any specific collection of queuers, since over the lifetime of the queue individual queuers are constantly being replaced. Although most of the discussion of ontological dependence concentrates on the specific (rigid) variety, generic dependence does receive some attention too, as for example in $[30,8,21,19,35]$.

Just as specific dependence can be generalised to generalised specific dependence, so generic dependence can be generalised to generalised generic dependence, in the obvious way:

(GGD) $\quad F$ s are generically dependent on $G \mathrm{~s}={ }_{\text {def }} \forall x(F(x) \rightarrow \square(\operatorname{Exists}(x) \rightarrow \exists y G(y)))$. 
This is, in fact, the primary form of generic dependence recognised in DOLCE [23], in which generic constant dependence of property $\phi$ on property $\psi$ is defined by the rule that at any time $t$ at which an instance of $\phi$ is present, some instance of $\psi$ must also be present (the quantification over times being the reason that this is called constant dependence, borrowing the terminology introduced in [35]). Given the definiens of (GGD), together with $F(a)$, the inference to the definiens of (GD) is immediate.

From the perspective of applied ontology, one reason why generic dependence is important is that it has been invoked, for example by Smith [33], to characterise the relationship between information artefacts and their concrete realisations in physical form. The existence of Pride and Prejudice, for example, is dependent on the existence of physical copies of that novel, but it is not dependent on the existence of any particular copy. This kind of example will be considered in more detail in $\S 5$. Thomasson [35] draws attention to the existence of chains of generic dependencies, as for example where a fictional character such as Sherlock Holmes is generically dependent on literary works (since the existence of the character of Holmes as a "cultural artefact" - Thomasson's words - is dependent on the existence of literary works in which he figures, any one of which would be sufficient for his existence), and the literary works themselves are generically dependent on their material copies, as already described.

The remainder of this paper is organised as follows. In $\S 2$, I look at some examples that have been cited as cases of generic dependence, and thereby show that there are a number of different ontologically significant varieties of this phenomenon. In $\S 3$ I discuss the problem of identity criteria for generically dependent entities, and why this is important. In the meat of the paper, $\S 4, \S 5$, and $\S 6$, I present detailed case studies of three kinds of putatively generically dependent entity, illustrating some of the problems discussed in the previous sections. Finally, $\S 7$ offers some concluding remarks by way of summary and pointers to possible future work. Sections 3 and 4 are closely based on material previously presented in [12].

\section{Some Varieties of Generic Dependence}

The definition of generic dependence states that an entity $a$ is generically dependent on entities of type $F$ so long as $a$ cannot exist without at least one $F$ existing, Quite apart from the concerns about the interpretation of "cannot" here, alluded to above, this general definition is broad enough to cover a range of distinctly different kinds of relationship. These form the subject of this section.

The first point to note is that the definition makes no explicit reference to time. Implicitly, though, it is natural to read the definition as stating that $a$ cannot exist at any given time without at least one $F$ existing at that time. The presumption, then, is that generic dependence is a synchronic relation: one does not expect to find such dependencies between entities existing at different times. In the case of specific dependence, however, Thomasson [35] distinguishes constant dependence, whereby $X$, say, cannot exist at any time without $Y$ also existing then, from historical dependence, whereby $X$ only depends on $Y$ for its coming into existence, but once in existence it can survive $Y$ 's extinction (thus a human being is historically, but not constantly, dependent on his or her parents). Does this distinction apply to generic dependence as well? I am certainly historically dependent on my actual parents, but since a child of any other parents could not have been me, it does not seem plausible to say that I am historically generically dependent on some parents or other: for persons, identity and ancestry seem to be intimately connected. On the other hand, perhaps not all types of entity are similarly constrained: a cake, for example, is historically dependent on the eggs which went into the dough, and we might be happy to say that the very same cake could have been made with different eggs, in which case the dependence would be generic rather than specific. ${ }^{1}$ Clearly this can only be definitively settled if we can agree on the identity criteria for objects such as cakes.

\footnotetext{
${ }^{1}$ This example is based on a suggestion from Claudio Masolo (personal communication).
} 
The second point to note is that the phrase 'at least one $F$ ' is compatible both with 'exactly one $F$ ' and 'more than one $F$ '. This suggests in the first instance that two rather different forms of generic dependence may be distinguished as follows:

1. Singular generic dependence: At each time that $a$ exists, there is a unique $F$ on which its existence depends, but this $F$ may be different at different times.

2. Multiple generic dependence: At any time at which $a$ exists there may be more than one $F$ supporting the existence of $a$.

Within the category of multiple generic dependence, two subtypes may be distinguished:

2a. Conjoint multiple generic dependence: At any time at which $a$ exists, its existence depends on a collection of $F \mathrm{~s}$, no proper subcollection of which is sufficient for $a$ to exist.

2b. Disjoint multiple generic dependence: At any time at which $a$ exists, there is a collection of one or more $F \mathrm{~s}$, any one of which is sufficient for $a$ to exist.

Examples of all three of these kinds can be found in the literature. Simons [30], for example, notes that 'a man cannot exist without carbon atoms, but which carbon atoms exist (and are part of him) are immaterial'. This is clearly a case of conjoint multiple generic dependence: the existence of one or two carbon atoms is not sufficient for a man to exist - there must exist trillions of them, but as Simons implies, exactly which trillions is immaterial. Of course, even the trillions of carbon atoms alone are not sufficient for the existence of the man: there must be atoms of other species as well, and all these atoms must be arranged in a highly specific way ${ }^{2}$; but some collection of trillions of carbon atoms is sufficient in the sense that they provide enough carbon for the man to exist, given the existence of sufficient atoms of other species. Consider now the total quantity of matter that goes to make up the man's body at a given time; this is a quantity of matter of a highly specific form and composition - let us call it a "viriform" portion of matter. ${ }^{3}$ Clearly a man cannot exist without there also existing a viriform portion of matter; and at any one time, the man's existence is determined by exactly one viriform portion of matter. At different times, however, the same man is determined by different viriform portions of matter. This is thus a case of singular generic dependence. ${ }^{4}$ As a consequence of the singular generic dependence of the man on viriform portions of matter, we can also say that the man is generically dependent in the same way on viriform collections of carbon atoms, by which I mean just those collections of carbon atoms which supply the carbon content of viriform portions of matter.

This example highlights a close relationship between singular generic dependence and conjoint multiple generic dependence. If an entity $x$ has conjoint multiple generic dependence on some entities of type $Y$, then there will typically be at any time some particular maximal collection $y$ of $Y$ s on all of which it depends at that time. Let the type of such collections be $Z$. Then $x$ has singular generic dependence on collections of type $Z$. In the example of the previous paragraph, $x$ is a man, $Y$ is the type 'carbon atom', and $Z$ is the type of viriform collections of carbon atoms. It may be going too far to claim that all cases of conjoint multiple generic dependence give rise to associated cases of singular generic inheritance in this way; and there is no reason to suppose that the converse holds in general. So this relationship, though in some cases close, is by no means invariable.

\footnotetext{
${ }^{2}$ Cf. Koslicki [17], who emphasises the important role of structure and arrangement in determining the identity of complex objects.

${ }^{3}$ From Latin vir, a man.

${ }^{4}$ This looks close to the notion of constitution, e.g., a man is constituted by a viriform portion of matter, and it seems plausible that when X constitutes $\mathrm{Y}$ then $\mathrm{Y}$ must be dependent on $\mathrm{X}$ in some way. Many cases of dependence, however, are not cases of constitution: as an example of how the two relations differ, note that whereas anything dependent on $\mathrm{X}$ is thereby also dependent on any proper part of X, nothing can be at the same time constituted both by X and by a proper part of X. Thus the man is dependent on, but not constituted by, a viriform collection of carbon atoms.
} 
A related case is where an object is dependent on a collection of objects of different types. An example is a broom, which requires a broomstick and a brush to exist, ${ }^{5}$ and thus may be said to be dependent on a two-element collection comprising a broomstick and a brush. Is this specific or generic dependence? This depends on what we say about preservation of identity under replacement of parts (as in the classic case of the Ship of Theseus). If I replace the brush of my broom and subsequently replace the broomstick, I certainly still have a broom; but do I have the same broom? If so, then the dependence of the broom on broomstick and brush is generic; if not, it is specific. It could be that the dependence is generic with respect to some members of the collection and specific with respect to others. A bicycle, for example, could be regarded as specifically dependent on its frame and generically dependent on wheels, handlebars, pedals, and so on, since the bicycle is identified by the serial number on the frame. In a similar way one might suggest that, in the age of transplant surgery, a human being is specifically dependent on its brain but generically dependent on all the other organ types.

The third kind of generic dependence, disjoint multiple generic dependence, is the one that will chiefly interest us in this paper. The prime exemplar, already alluded to, is the relation between an information artefact and its physical bearers. A poem, for example, may be considered to be an information artefact. ${ }^{6}$ The physical realisations of a poem may take many forms, including written or printed texts, magnetic or optical storage in digital form, performances in which the poem is recited aloud, and neurological traces in the brains of people who have memorised it. Many such physical realisations can exist simultaneously, any one of which would suffice on its own for the poem to exist; or, to put it differently, there can be many different copies of an information artefact, a point stressed by Smith [33]. This is in stark contrast to both singular and conjoint multiple generic dependence, for both of which the notion of "copy" is inapplicable. The different viriform portions of matter that support the existence of a man at different times are not different copies of the man, nor are the individual carbon atoms that contribute to the man's existence copies of anything, certainly not the man (and if in a Platonist spirit one says that each is a "copy" of the Form of Carbon, then this is beside the point since the generically dependent entity under consideration is not the element carbon but a man).

As far as I am aware, the distinctions drawn in this section between different varieties of generic dependence have not appeared in the literature up to this point, and yet they seem to indicate that the broad notion of generic dependence covers what are really very different forms of relationship. Perhaps one reason for the neglect of these distinctions is the difficulty of expressing them using the same sort of logical resources that have been used for expressing the more general concept, as illustrated by (GD) and (GGD) above. For those definitions merely establish a connection between the existences of one thing and another; it is not obvious how to extend them so as to include reference to the nature of the connection between the things themselves. Thus instead of just saying that this man cannot exist without some viriform portion of matter existing, one would like to say that somehow the man is made from the matter.

\section{Generic Dependence and Identity}

With all forms of generic dependence, there is a one-to-many relationship between the dependent entity and the entities on which it depends - in the terminology of Correia [5], these are its 'dependees'. This means that we can have different dependees corresponding to the same dependent - in the case

\footnotetext{
${ }^{5}$ In the words of Wittgenstein $[37, \S 60]$, 'if the broom is there, that surely means that the stick and brush are there'.

${ }^{6}$ I make no attempt here to define "information". The sense intended here is situated somewhere between the very broad "syntactic" notion covered by Shannon and Weaver in their Mathematical Theory of Communication [29], and the highlyfocussed "semantic" notion advocated by, for example, Floridi [9], according to which only true propositional content counts as information. Thus for me poems, pictures, and pieces of music may count as information artefacts even if they lack propositional content, or if such propositional content as they contain is in fact false. Cf., Sloman [31]: 'It is important not to restrict the notion of "information" to what is true or even to whole propositions that are capable of being true or false'. This is in agreement with the notion presented by Smith [33]
} 
of singular generic dependence, a dependent can have only one dependee at a time, but in the case of multiple generic dependence, a dependent can have many dependees simultaneously. The key word here is 'same': in virtue of what do different dependees support the existence of the same dependent? Or, in other words, what are the criteria for identity of generically dependent entities?

The word 'same' points to the existence of an equivalence relation. Any relation of the form ' $x$ is the same as $y$ in respect of $R$ ' should be reflexive, symmetric, and transitive if it is to support any kind of identity statement. In particular, this should go for the relation between dependees of the same dependent: in other words, ' $x$ and $y$ are dependees of the same dependent' should be an equivalence relation. If this is the case, then there will be a strict one-to-one correspondence between dependent entities and equivalence classes of dependees: to each dependent can be uniquely assigned the class comprising all its dependees. In mathematics, a typical move at this point would be to identify the dependent entity with that equivalence class. A mathematical example is the notion of a rational number $\frac{p}{q}$, which can be identified with the class $\left\{\langle x, y\rangle \in \mathbb{Z}^{2} \mid y \neq 0 \wedge x q=y p\right\}$ of all ordered pairs of integers in the same ratio as $p: q$. This has the virtue of parsimony even though it may go against the grain of our intuition - arguably, one can have a perfectly serviceable intuition of rational number that is quite distinct from the notion of a class of pairs of integers. ${ }^{7}$ None the less, with or without this identification, it is arguable that unless these equivalence classes can be picked out, a properly constituted notion of a generically dependent entity is lacking.

Frege [10] drew attention to a group of concepts $X$ for which the notion of $X$ itself is logically dependent on a prior notion which, once the concept $X$ is available, it is natural to express using the words 'has the same $X$ as'. This latter notion is an equivalence relation which can be defined without any reference to the concept $X$ itself. Frege noted that the concepts of number, direction, and shape can all be derived in this way.

In the case of (cardinal) number, the relevant relation is defined as follows:

- Set $X$ has the same number as set $Y$ if and only if there is a bijection ${ }^{8}$ between the elements of $X$ and the elements of $Y$.

Notice that bijections can be defined without reference to number; but on the other hand, according to Frege's argument, number cannot be defined without having the prior notion of same number to establish an identity criterion for the new concept. Thus same number is shown to be logically prior to number. Instead of 'has the same number as', the term 'equipollent' can be used. ${ }^{9}$ Number is now defined in terms of equipollence as follows: The number of set $X$ (that is, the number of its elements, its cardinality) is the set of all sets equipollent to $X .{ }^{10}$ This set is what would now be recognised as an equivalence class under the equipollence relation. ${ }^{11}$

Similarly, direction is logically dependent on the relation has the same direction as, which is routinely expressed as 'is parallel to'; and shape is logically dependent on has the same shape as ('is geometrically similar to'). In particular, the shape of an object can be defined as the equivalence class comprising all objects which have the same shape as it. This kind of definition works well so long as (a) a domain of "objects" $\mathfrak{D}$ is established for the relation has the same $X$ as to be defined on, and (b)

\footnotetext{
${ }^{7}$ An even worse case is the concept of real number. Mathematically, a real number can be identified as an equivalence class of convergent sequences of rational numbers under the "co-convergence" relation — but again, this is remote from the intuition, such as it is, that most people form of real numbers.

${ }^{8}$ That is, an exhaustive one-to-one correspondence.

${ }^{9}$ Frege's term was gleichzahlig i.e., "equal-numbered".

${ }^{10}$ Frege did not himself formulate this in terms of sets: he spoke of the number which belongs to the concept $F$ (die Anzahl, welche dem Begriffe $\mathrm{F}$ zukommt) and equated this to the extension of the concept "equipollent to the concept F" (der Umfang des Begriffes "gleichzahlig dem Begriffe F”).

${ }^{11}$ This is not unproblematic: What set is this relation defined on? Frege supposed this could be the set of all sets, but as Russell pointed out to him, this notion can lead to devastating paradoxes. An adequate discussion of this point would take us well out of scope of this paper.
} 
within domain $\mathfrak{D}$, has the same $X$ as can be defined as an equivalence relation in a way that does not make use of any prior concept of $X$.

This strategy is applied in the first instance to cases of logical dependence of one concept on another (e.g., rational number on integer, integer on set, direction on line, and shape on figure or physical object). Of interest to us here is whether such cases also exemplify ontological dependence between the entities themselves: are rational numbers ontologically dependent on integers, integers on sets, and so on? If so, then all of these will be cases of disjoint multiple generic dependence, since in every case the dependent object will correspond to (or be identified with) a class containing many different simultaneously existing dependees.

There is a problem here, however. According to the definitions presented above, an $x$ is generically dependent on $F$ s so long as $x$ cannot exist unless at least one $F$ exists. But in the case of mathematical entities such as numbers, lines, sets, and geometrical figures, it is generally held that, if they exist at all, they exist necessarily, and the simple definitions of dependence imply that anything whatever is dependent on any entity that itself exists necessarily. It was precisely to rule out this kind of spurious dependence that Simons [30] introduced an extra clause in his versions of the definitions of dependence to ensure that dependees are not necessary existents. But if necessary existents are ruled out as the objects of ontological dependence, then nothing can ever be said to be ontologically dependent on numbers, pairs of numbers, sets of pairs of numbers, and other such necessarily existent entities. It may be that Fine's essentialist conception of dependence (obtained by reading the $\square$ in the definitions as $\square_{x}$, as explained in §1) can circumvent this difficulty, but since in any case the main focus in what follows is on cases in which the dependees are not necessary existents, I set this point aside here.

It is, therefore, not unreasonable to hope that the same Fregean approach using equivalence classes might be serviceable in establishing identity criteria for generically dependent entities whose dependees are not necessary existents. In the next three sections I will present a series of case studies of types of entity for which there is at least a prima facie case for regarding them as generically dependent, in order to see to what extent this prima facie appearance can be upheld in the light of considerations of identity.

\section{Case Study I: Shapes}

Shapes provide a particularly good testing-ground for the ideas presented in $\S 3$. I begin by assuming unquestioningly that there is a prima facie case for regarding shapes as generically dependent entities. In the light of the discussion ensuing from that assumption, I go on to consider whether the assumption can be upheld.

Figure 1 shows five instances of a conventional heart shape - incidentally illustrating the independence of shape from size and orientation. It is quite natural to say that the five figures all have the same shape. This way of speaking, if taken literally, implies that there is something, the shape, that they all have. The shape stands in a certain relation to each of the five exemplars in the figure, ${ }^{12}$ a relation we might express in the form ' $X$ is the shape of $Y$ '. What has the shape, in these particular instances, are certain quantities of black ink arranged on the paper in such a way as to contrast clearly with the white background, although other exemplars could exist having different physical constitutions, for instance solid patches of red paint, or cardboard cut-outs. The shape might be said to exist only because some such exemplars exist, and for this reason it is natural to say that the shape is generically dependent on exemplars. ${ }^{13}$ On the Fregean view, this generically dependent thing is identified with the class of all its exemplars. We do not have to go along with that construction, but we must surely at least go halfway to meet Frege in acknowledging that the possibility of collecting these exemplars together as instances of

\footnotetext{
${ }^{12}$ It should be noted that each separate copy of this paper supplies another five distinct exemplars of this shape.

${ }^{13}$ Of course it might be possible to mathematically specify shapes which happen to have no physical exemplars in this world. If by shape is meant something physical then such mathematically-specified but uninstantiated shapes must be regarded as existing merely possibly, and not in actuality.
} 
some one common shape depends on the existence of some equivalence relation which holds between each pair of exemplars. I now turn to the task of defining that relation.

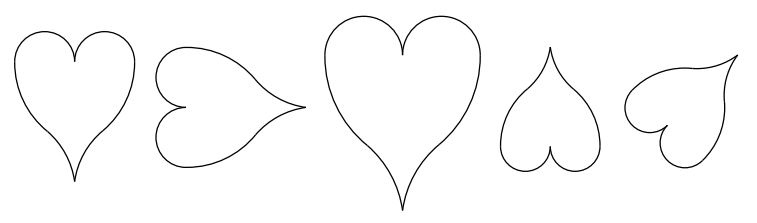

Figure 1: Five realisations of the same shape

For Frege, the same-shape relation is glossed as 'is geometrically similar to'. Geometrical similarity, however, is first and foremost defined as a relation on abstract geometrical objects; these may be understood, in standard mathematical fashion, as subsets of $\mathbb{R}^{n}$, for some $n \in \mathbb{Z}^{+}$. But the physical exemplars of the heart shape shown in Figure 1 are not subsets of $\mathbb{R}^{n}$ for any $n$ : they are portions of black ink arranged on white paper. It is therefore necessary to ask in what way this relation can be applied to the very different domain of physical objects.

\section{Similarity of geometrical objects}

In defining what is meant by geometrical similarity as it applies to objects in geometrical space, the key notion is that of distance, which serves as a measure of the separation between two points. Writing $\Delta(p, q)$ for the distance between points $p, q \in \mathbb{R}^{n}$, defined by the usual Pythagorean rule, we have: ${ }^{14}$

Definition of geometric similarity between figures in Euclidean space. Two subsets $X, Y \subseteq \mathbb{R}^{n}$ are geometrically similar if and only if there is a bijection $\phi: X \rightarrow Y$ such that, for some constant $\kappa \in \mathbb{R}^{+}$, the following relation holds:

$$
\forall x, x^{\prime} \in X . \Delta\left(\phi(x), \phi\left(x^{\prime}\right)\right)=\kappa \Delta\left(x, x^{\prime}\right) .
$$

In other words, distances between points in $X$ are multiplied by a constant factor $\kappa$ when the points are mapped by $\phi$ into their images in $Y$. This is straightforward and familiar; the important point for us is that the relation thereby defined is an equivalence relation, for this is what enables the Fregean move by which a figure's shape is identified with the equivalence class of all figures geometrically similar to it.

\section{Similarity of physical objects}

When we turn from $\mathbb{R}^{n}$ to the physical world, the matter is less straightforward. Whereas distance in $\mathbb{R}^{n}$ can be defined mathematically, in physical space the notion of distance is inextricably tied up with that of measurement, and the key fact about measurement here is that all measurement has finite precision. This means that whereas in $\mathbb{R}^{n}$ the space of possible distances is simply $\mathbb{R}^{+} \cup\{0\}$ (since distances can be arbitrary non-negative real numbers), the space of measured distances in physical space cannot take this form. To see this, it suffices to reflect that it is not meaningful to ask whether the length of a rod in metres is rational or irrational.

\footnotetext{
${ }^{14}$ Here I am only dealing with Euclidean space - complications arise when non-Euclidean spaces are considered. For example, on the surface of the sphere, triangles cannot be similar without also being congruent. This is because, in this space, the sum of the interior angles of a triangle exceeds $2 \pi$ by an amount that is proportional to the area of the triangle, and hence no triangle can be expanded or contracted without changing shape. Only in Euclidean space is shape entirely independent of size.
} 
Given that in physical space distances can only be characterised in terms of measurement, and that measurement always has a finite precision, corresponding to the resolving power of the measuring instrument, it follows that any analogue of geometrical similarity for physical objects can only be defined relative to a specified level of resolution. Consider two objects whose shapes are to be compared, say $P$ and $Q$, where without loss of generality it may be assumed that $Q$ is at least as big as $P$. Suppose the volume of $P$ is $v$ and that the resolving power of the best available measuring instrument is such that the smallest distance that can be distinguished is $h$ (I shall describe this as 'resolution $h$ '). Then in principle, within the physical space occupied by $P$ it is possible to distinguish, say, $n \approx v / h^{3}$ points, and to each pair $x, y$ of these points can be assigned a distance $\Delta_{h}(x, y)$ that is some multiple $k h$ of the minimum discernible distance. ${ }^{15}$ Let $S_{h}(P)$ be this set of $n$ discernible points in $P$; these could, perhaps, be thought of as "blobs" of diameter $h$, though it is not really possible to assign any definite shape to these blobs.

To say that objects $P$ and $Q$ have the same shape is to say that the points discernible in $P$ at resolution $h$ can be mapped onto some set of points discernible in $Q$ at that resolution, such that, first, the distances between pairs of the latter set of points are not discernibly different, at resolution $h$, from some constant multiple of the distances between the pairs of points from the former set to which they correspond under the mapping; and second, every point in $Q$ discernible at resolution $h$ is "sufficiently near" the image of some discernible point in $P$. In other words:

Definition of same shape for physical objects. Physical objects $P$ and $Q$ (where $Q$ is at least as big as $P$ ) have the same shape, at resolution $h$, if, for some constant $\kappa \geq 1$, the set of points discernible in $P$ at resolution $h$ can be mapped into the set of such points of $Q$ by means of an injective mapping $\phi: S_{h}(P) \rightarrow S_{h}(Q)$ such that the following relations hold:

$$
\begin{aligned}
& \text { 1. } \forall x, y \in S_{h}(P) \cdot\left|\Delta_{h}(\phi(x), \phi(y))-\kappa \Delta_{h}(x, y)\right| \leq h \\
& \text { 2. } \forall x \in S_{h}(Q) . \exists y \in S_{h}(P) \cdot \Delta_{h}(x, \phi(y)) \leq \kappa h
\end{aligned}
$$

This is perhaps as near as it is possible to come to the notion of geometric similarity in a physical setting, in which the idea of "exact distance" gains no purchase.

An immediate consequence of this is that a pair of objects with the same shape at one resolution may have different shapes at a finer resolution. For physical objects, sameness of shape is inescapably tied to the level of resolution at which the objects are examined; and since, according to the Fregean argument, shape is logically dependent on sameness of shape, it follows that, for physical objects, shape is also tied to levels of resolution. This is, of course, a familiar idea in Computer Science, where the notion of resolution, which I have handled in a very crude manner here, has been considerably refined, for example in the technique of multiscale representation in which a series of images at different resolution levels can be obtained by convolving an original image with Gaussian kernels of different variances (see $[6$, Ch.7]).

Unfortunately, the Fregean construction cannot be achieved in this instance, because the same shape relation on physical objects, as defined above, is not an equivalence relation. It is perfectly possible to have three objects $\mathrm{A}, \mathrm{B}$, and $\mathrm{C}$, such that, at some resolution $h$, A has the same shape as $\mathrm{B}$ and $\mathrm{B}$ has the same shape as $\mathrm{C}$, but A does not have the same shape as $\mathrm{C}$. This is essentially because sameness of shape, as here defined, does not capture a notion of identical shape so much as a notion of indiscernible (at resolution $h$ ) shape; and it is a familiar fact that, unlike identity relations, indiscernibility relations are not transitive. The crucial implication of this, for us, is that there is no coherent notion of "exact shape" for a physical object, only that of objects being more or less approximately the same shape as other objects.

\footnotetext{
${ }^{15}$ This is somewhat oversimplified since in practice the resolution of our observations will not harmonise with the levels of resolution available in the system of units used for recording them - e.g., given resolving power (for lengths) of $0.03 \mathrm{~mm}$, recording to 1 decimal place is too coarse and recording to two decimal places is too fine. For an attempt to deal with this issue in an ontological framework, see [25].
} 


\section{Similarity between physical and geometrical objects}

It is often said that the shapes of physical objects can also approximate to the shapes of ideal geometrical objects (compare [27]). Thus there are many approximate spheres in the physical world, but no geometrically exact spheres, the exact sphere being an inhabitant of mathematical, not physical space. This is true so far as it goes, but what is meant here can be more carefully explained using resolution-based shape comparison. An explanation is needed since on the face of it the idea of comparing something physical with an abstract mathematical construction is paradoxical: the two belong to such entirely different realms that any notion of comparison might seem to be out of the question.

If the shapes of, say, a sheet $P$ of A4 paper and a certain rectangle $R$ defined in $\mathbb{R}^{2}$ are to be compared, then some way of matching up points in the former with points in the latter is needed. There are, at least on the orthodox view, uncountably many points in $R$, but there does not seem to be any meaningful sense in which uncountably many points can be attributed to $P$. There is already something dubious about the idea of attributing infinitely many points to $P$, since as noted earlier, $P$ can only be observed at all at some finite level of resolution, and at any such level only finitely many points can be distinguished within it. One might, of course, entertain the notion that, if there is no limit to how fine the resolution level can be, then there is no limit to how many points can be discerned in $P$, so that the number of points in $P$ is, if not actually infinite, at least potentially infinite. But there is no good reason to believe that, in the physical world, resolution could be made indefinitely fine. On the contrary, in the state of our current understanding of physics, the Planck length (approximately $1.6 \times 10^{-35} \mathrm{~m}$ ) is believed to provide a lower bound for the resolution of any possible technique of measurement.

What, then, does it mean to say that a piece of paper, considered at resolution $h$, has the shape of a rectangle with sides in a given ratio? The definition of strict geometric similarity for geometric figures cannot be used here, nor can the definition of sameness of shape at resolution $h$ given for physical objects. Instead, the criterion must be modified by combining elements from the two definitions as follows:

Definition of a physical object having the same shape as a geometrical object. At resolution $h$, a physical object $P$ has the same shape as a geometrical object $Q \subset \mathbb{R}^{n}$ if there is an injective mapping $\phi: S_{h}(P) \rightarrow Q$ from the set of points discernible in $P$ at resolution $h$ into $Q$ such that, for some constant $\kappa>0$ :

$$
\begin{aligned}
& \text { 1. } \forall x, y \in S_{h}(P) . \Delta(\phi(x), \phi(y))=\kappa \Delta_{h}(x, y) \\
& \text { 2. } \forall x \in Q . \exists y \in S_{h}(P) \cdot \Delta(x, \phi(y)) \leq \kappa h .
\end{aligned}
$$

The point here is that the objects in the pure geometric world, being given to us by thought rather than by observation, can be specified with infinite precision: in particular, the distances between points in such figures can be arbitrary real numbers and are not constrained to being multiples of some minimal discernible distance. ${ }^{16}$

\section{Are shapes really generically dependent entities?}

The foregoing discussion has shown how it is possible to use locutions of the form ' $x$ has the same shape as $y$ ', where $x$ and $y$ can be geometrical figures or physical objects. When at least one of them is a physical object, the assertion has to be supplemented, at least implicitly, by a qualification of the form 'at resolution $h$ ' (in practice, this might be phrased somewhat loosely, e.g., 'as nearly as I can tell' -

\footnotetext{
${ }^{16}$ It is perhaps worth commenting here that since distances in mathematical space are pure numbers, whereas distances in physical space are expressed as multiples of some physically defined unit of measurement, it would not make any sense to compare the sizes of mathematical and physical objects; the reason we can compare their shapes is that the relevant quantities in each case are not distances but ratios of distances, and these are always pure numbers.
} 
meaning, at the finest resolution I am capable of). But because, in all cases other than where $x$ and $y$ are both geometrical figures, has the same shape as is not an equivalence relation, it is not possible to define generically dependent shapes with the property that two objects can be said to have the same shape just when they are exemplars of the same generically dependent shape. For the failure of transitivity in the same shape relation means that there can be a sorites series of objects such that each object has the same shape as its neighbours but the objects at the beginning and end of the series do not have the same shape. This calls into question whether shapes can be regarded as generically dependent objects after all.

What is the alternative? On the generically dependent view, the logical form of the statement that $x$ has the same shape as $y$ at time $t$ must be something like this:

$$
\exists s(\operatorname{HasShape}(x, s, t) \wedge \operatorname{HasShape}(y, s, t)) .
$$

Here the variable $s$ ranges over generically dependent shape entities, and HasShape denotes the relation in which each exemplar of a shape stands to the shape itself, the latter being generically dependent on exemplars of that kind. But modern information systems ontologies such as BFO [16] and DOLCE [23] do not take this line; instead, they treat an object's shape as something that is specifically dependent on that object, meaning that the shape belongs uniquely to that object and cannot be shared with any other. In DOLCE, shapes, along with such things as colours, volumes, weights, and densities, are classified as qualities. The identity of an object's shape is tied to the identity of the object itself: the shape comes into existence when the object comes into existence, and endures for as long as the object does. This does not mean that an object cannot change shape, though; what happens, according to DOLCE, is not that the object assumes a different shape, but that the object's shape assumes a different value. The values that may be assumed by a quality are entities of another kind, called qualia, which collectively constitute a domain known as a quality space - in the case of shape, one can speak of shape qualia in shape space. These quality spaces are similar to the conceptual spaces of Gärdenfors [13].

On this picture, variability of shape shows up as a time-dependency, not of the shape on its bearer, but of the value of the shape on the shape. Writing shape $(o)$ to refer to the shape which uniquely inheres in the object $o$, formula (SS1) must be replaced by

$$
\operatorname{value}(\operatorname{shape}(x), t)=\operatorname{value}(\operatorname{shape}(y), t) .
$$

The shape-as-quality view embraced by DOLCE has a solid pedigree in the Aristotelian fourcategory ontology encapsulated in the ontological square [20,28], which presents a cross-classification of the entities of an ontology along the dimensions of universal vs particular (distinguishing types from their instances) and substance $v s$ accident (distinguishing independent from dependent entities). Thus the roundness of this ball is an accidental particular inhering in (and thus dependent on) the substantial particular this ball; and these two particular entities are instances of the accidental universal roundness and the substantial universal ball respectively. The ball itself is said to exemplify roundness.

The roundness of this ball is not quite the same as the shape of the ball conceived as a quality in DOLCE. The former is a trope, a specific instance of a property inhering in an object [1]. In DOLCE terms, the property in question is not just a quality but a quality's having a particular value. Thus a trope could be regarded as a quality/value pair. If the quality changes value (as when an object changes shape), then the previous trope is superseded by a new one. When the value of some quality changes continuously, there is a continuous succession of different values for that quality, and hence a continuous succession of tropes.

Even under this trope-like view of shape, our earlier worries about identity persist. Although the shape of an object, as a specifically dependent entity unique to that object, is no longer subject to identity problems (except insofar as such problems may arise in connection with the object itself), these problems are now shifted into the quality space which supplies values for the shape. The ontological status of these values is far from clear. They do not appear to be generically dependent entities themselves: to take a 
clear-cut case, consider the quality-type length. The space of values that may be assumed by individual instances of this quality (that is, the lengths of individual objects) must include not only the values actually assumed by the lengths of existing objects, but also all the possible values that these lengths, as well as the lengths of non-existent but possible objects, could assume. These values are not dependent on any concrete entities possessing them; they seem to be entirely abstract, as is recognised by ontologies such as DOLCE which provide a category of abstracta to put them in. If these values are exact, then no single one of them can be assigned to any specific quality instance, but rather a range with indeterminate boundaries; and it is these rather indeterminate ranges which must play the role of length - or shape - qualia. And such ranges suffer from the same identity problems as the generically-dependent shapes considered earlier.

To conclude this case study, then, it would appear that there are serious difficulties with the view that, outside the realm of pure mathematics, shapes exist as generically dependent entities. The considerations that led to this conclusion hinge crucially on the role played by measurement in determining the shapes of physical objects; similar arguments could be brought to bear on a range of other measurable properties, such as colour, mass, temperature. With most of these, however, there is less of an initial temptation to regard colours, masses, and temperatures as generically dependent entities, probably for the reason that the corresponding quality spaces are of low finite dimension, whereas the dimensionality of shape-space seems to be infinite.

I turn next to another class of entities with a prima facie case for being regarded as generically dependent, and consider whether that claim is on equally shaky ground.

\section{Case Study II: Words, Texts, and other Linguistic Entities}

In the case of linguistic entities, it is natural to see the well-established dichotomy between types and tokens as aligning with the distinction between generically-dependent entities and the things they depend on, respectively. Natural though this may be, it should not be accepted it uncritically, and as will become apparent below, there may be reasons to doubt whether the relation between type and token is always precisely that of dependant to "dependee". The terminology of 'type' and 'token' is due to C. S. Peirce; for a detailed philosophical discussion, and pointers to further references, see [36].

In $\S 4$ there are numerous occurrences of the word 'shape'. These are not tokens; the tokens are rather the physical realisations of these occurrences in individual copies of this paper. In the case of printed copies, these tokens take the form of patterns of ink on paper; in electronic copies, they are patterns of bits in computer storage. If the text is read aloud, there are ephemeral tokens in the form of patterns of sound-waves. Arguably, the patterns of neural firing that are activated in the brain of someone reading or hearing the paper read are also tokens; and so again might be whatever part of the brain's neurological organisation underlies the fact that the individual in question has knowledge of the word 'shape'.

In this description I have several times used the word 'pattern' to describe the tokens, but it is worth considering whether this is right. It is certainly consistent with the view of information entities promoted by Smith [33], who states that what an information artefact, considered as a generically dependent entity, depends on is not material objects per se but rather patterns exhibited by material objects. These patterns are specifically dependent on the physical objects in which they inhere. On this view, then, the word 'shape', when manifested in printed form, does not depend directly on a certain portion of ink on paper but rather on the pattern in which that portion of ink is arranged - where pattern here must be understood to be the unique, non-transferrable pattern-instance inhering in that portion of ink. Since this pattern itself depends on the ink, it could at least be said that the word is indirectly generically dependent on portions of ink by virtue of being directly dependent on patterns inhering in the ink.

For the putative alignment of dependant/dependee with type/token, does it make any difference whether a word is said to depend on something material or on a pattern-instance inhering in something material? This depends on whether tokens are taken to be physical things or pattern-instances. Here 
I shall assume what I take to be the orthodox view of this, as expressed by Wetzel [36]: 'tokens are concrete particulars, composed of ink, pixels of light (or the suitably circumscribed lack thereof) on a computer screen, electronic strings of dots and dashes, smoke signals, hand signals, sound waves, etc.'. In the light of the phrase in parentheses, the requirement that tokens should be material must be modified to the somewhat weaker requirement that they should be physical, since the relevant patterninstance can be borne by an absence of material as easily as by a portion of material, so long as the limits of that absence are marked by the presence of material. Thus a hole can be a token. But this is not a serious problem; a hole is a non-material physical particular that is specifically dependent on the matter constituting its material host. What matters, for something to play the part of a token, is that it is physical; it may be material but it does not have to be.

I am not aware of any critical issues which depend on the point currently under discussion. At least in the case of words, a type can be said to depend on a pattern instance, which in turn depends on a physical particular; dependency being transitive, the type depends also on the physical particular. To put it somewhat differently, the type depends on the physical particular by virtue of that particular's manifesting a certain pattern. Although this may seem a minor modification, it leads us to a fruitful way of describing linguistic entities.

In semiotics, language is said to exhibit double articulation, a term introduced by Martinet [22]. The idea is that on the one hand there are words, which are meaningful units (leaving aside the considerable theoretical difficulty in defining exactly what a word is), and on the other hand, these words are built up out of smaller, individually meaningless units - phonemes in the case of spoken language, and letters, or graphemes, in the case of written language. What is it about the pattern exhibited by a particular portion of ink in virtue of which that portion is a token of the word 'shape'? The obvious answer is that the ink consists of a linearly arranged sequence of smaller portions which respectively exhibit patterns in virtue of which they are tokens of the letters 's', ' $h$ ', 'a', 'p', and 'e'. One way of describing this is to say that the physical token bears content at two distinct levels: its graphemic content is the letter sequence ['s', 'h', 'a', 'p', 'e'], and its verbal content is the word 'shape'; and it bears that verbal content by virtue of bearing the graphemic content. Not only that, of course: graphemic content alone is not sufficient to determine verbal content, since in different contexts the same graphemic content may determine different verbal contents, as for example the grapheme sequence manifested in 'estate' corresponds to different words depending on whether the context determines that it should be read as an English word or an Italian one. ${ }^{17}$

This notion of levels of content can be extended further. A written or printed token of the word 'shape' has a certain visual content: this is the visible pattern exhibited by the physical token. It is by virtue of possessing this visual content that it also possesses graphemic content, that is, it can be interpreted as a sequence of letters. Again note that the graphemic content exists not by virtue of the visual content alone, but the visual content together with its context - the visual content of 'CCCP', for instance, gives rise to different graphemic contents depending on whether the token that bears that content appears in a document written in the Roman alphabet or the Cyrillic. Of course not all visual contents support graphemic content; with language, intention plays a central role, and normally a token with a particular visual content would only be said to bear some graphemic content if there is reason to believe that the token was produced with the intention of conveying that graphemic content (and any further content that depends on it).

As already said, printed or written tokens may bear verbal content by virtue of having some graphemic content, in an appropriate context. At the level of verbal content, individually meaningful units are strung together to form a sequence. Above the level of verbal content one may consider, for example, the propositional content, which is borne by a sequence of words forming a sentence - here I do not restrict this to propositions in the sense of bearers of truth-value, but include also questions, commands,

\footnotetext{
${ }^{17}$ In the present context, of course, neither of these is the case since the grapheme sequence is being exhibited as just that, a grapheme sequence with no verbal content.
} 
wishes, promises, and so on. Context-dependence remains in force here, of course: which proposition is conveyed by a particular sequence of words may depend on the context in which it occurs, and everyone is familiar with cases of ambiguity where the context is insufficient to decide between two or more interpretations of a sentence - to decide, that is, which of two or more possible propositional contents can be ascribed to a token sentence by virtue of its verbal content.

In the case of spoken language, a similar pattern appears, except that now the nature of the tokens, the physical content-bearers, is different. They are occurrents rather than continuants, trains of sound waves emanating from a speaker (whether human or mechanical). The sound-pattern borne by the physical disturbance in the air constitutes the auditory content of the token. Depending on context, the token may also possess, by virtue of its auditory content, phonemic content, meaning that it may be interpreted as a sequence of phonemes. And by virtue of possessing that phonemic content, it may, if the context is suitable, also possess verbal content - and thence, as in the written case, propositional content.

The relationship between levels, whereby a token may possess content at one level by virtue of possessing content at a lower level, is readily described in terms of dependence. Thus the word 'shape', as a generically dependent entity, depends on the existence of tokens which, by virtue of their form and context, are bearers of this particular verbal content, making them instances of that word. Each such instance is an instance by virtue of possessing a particular content at the next lower level — which may be a sequence of letters, an idiogram, a sequence of sounds, or a sequence of bytes in electronic storage. Thus a word type, as a unit of verbal content, can be described as generically dependent on types at a lower-level, these lower-level types being sequences of units of graphemic, phonemic, or digital content. And each of these is generically dependent on types at a lower level still: these are the visual, auditory, or electronic contents whose individual instances inhere, as specifically dependent particulars, in something physical. That physical something, the token, is the ultimate bearer of all these levels of content.

If this story is to be upheld, then none of these generically dependent items could exist in the absence of tokens. Is this true? Partly, this may depend on what is allowed to count as a token. As already suggested above, in addition to the kinds of tokens discussed in the last few paragraphs, there is a case for also including the neurological configurations in virtue of which a person has knowledge of particular letters, phonemes, words, sentences, etc. If these are not included as tokens, then a curious consequence follows. Consider a language which has never been written down. Outside human brains, the only tokens of its words and sentences are spoken ones. In particular, at a time when a given word is not being uttered by any of the speakers of the language, it has no non-neurological tokens, so unless neurological tokens are counted as equally good bases for generic dependence, one would have to say that the word ceases to exist between its successive utterances; and this seems rather implausible. Granted, then, that neurological tokens can support the existence of corresponding types, is it now true that a type cannot exist without any of its tokens existing? If all records of a certain word, whether inscribed in stone, printed on paper, stored on computer discs, or remembered by brains, are destroyed, then it is reasonable to say that the word has passed out of existence. No subsequent word that comes into existence, however similar in form or meaning, can be identified as the same word as the old one; the question remains whether any linguistic item can be said to exist in the absence of tokens.

The only area where I can find plausible examples of this is mathematics. There is a certain very large integer called Skewes' Number, equal to $10^{10^{10^{34}}}$, which the mathematician G. H. Hardy is reported to have described as 'the largest number which has ever served any definite purpose in mathematics'. Now consider the decimal numeral for Skewes' number. This numeral can be specified precisely as a sequence consisting of a ' 1 ' followed by $10^{10^{34}}$ zeroes. It seems strange to say that this sequence (the numeral, that is, as opposed to the number itself) does not exist. Yet it is quite certain that there is not, and never has been, any token of this numeral, for the number of zeroes needed is far larger than the number of elementary particles in the observable universe. So this looks like a case where the type can be said to exist without having any tokens. But this does not mean that this tokenless numeral is floating in some platonic mode of existence completely detached from concrete reality, since there is an exact 
and fully tokened description that would have to be satisfied by any of its tokens, were they to exist.

In $\S 3$ I indicated the importance of identity for generically dependent entities, and in $\S 4$ this was shown to be problematic in the case of physical shapes, casting doubt on their viability as generically dependent entities. How do the linguistic entities discussed in this section fare in this respect?

In the case of graphemic content the situation is for the most part clear. The system of graphemes of any language form what Haugeland [15] called a digital system. For Haugeland, one of the hallmarks of a digital system is the possibility of $100 \%$ copying fidelity. He defined a digital system rather generally as 'a set of positive and reliable techniques (methods, devices) for producing and reidentifying tokens, or configurations of tokens, from some prespecified set of types', where a 'positive' technique is 'one that can succeed absolutely, totally, and without qualification'. Graphemes are an example of tokens that can be positively and reliably produced and reidentified, which is why $100 \%$ copying fidelity is possible for graphemic content. For any kind of digital content, any piece of content of that kind can be broken down into units, that is, minimal content elements which cannot be analysed further into smaller elements of the same kind. Thus the units of graphemic content are individual graphemes, and the graphemic content of a token consists of a sequence of graphemes. Two tokens have the same graphemic content so long as those contents consist of the same graphemes in the same order. That they do so is an absolute judgment, not dependent on any analogue of a level of resolution. This does not mean that it is always easy to discern the graphemic content of a token — we all know people whose handwriting is illegible — but this is a separate issue.

To clarify this point, note that visual content is not governed by a digital system, and precisely because it is visual it is dependent on resolution (visual acuity). This means that one could have a sorites series of tokens running from, say, something indistinguishable from ' $\mathrm{A}$ ' to something indistinguishable from 'B', in which all pairs of neighbouring items are indistinguishable. Thus visual content is like shape (and indeed in many ways closely akin to it) in lacking robust criteria of identity. But any attempt to assign graphemic content to all the tokens in the series is bound to fail: at the beginning of the series there are clear tokens of 'A', at the end of the series there are tokens of 'B', while in the middle of the series the items are not tokens of any letter - certainly not letters that are 'intermediate' between 'A' and 'B', because there is no such thing. Admittedly, this is a somewhat artificial exercise, since in reality graphemes usually occur in the context of words, and the context can often enable one to recognise as, say, a token of 'A' something which in isolation it would be impossible to assign a letter to. The key point here is that because graphemes form a digital system, there cannot be a sorites series of graphemes running from 'A' to 'B', whereas there can be such a series of visual appearances. The fact that graphemes can be copied with $100 \%$ fidelity means that any two tokens to which graphemic content can be ascribed must have either the same or different graphemic contents, and crucially, the relation has the same graphemic content as is transitive, indeed an equivalence relation. Although this account is somewhat idealised, it does point to an important distinction between graphemic and visual content - and perhaps significantly, the former, but not the latter, may be classed as linguistic in nature.

Much the same applies to verbal content. Sometimes, it is true, it may be unclear whether two grapheme-sequences should count as the same or different words. Is 'jail' the same word as 'gaol'? They agree as regards pronunciation, meaning, and etymology; the only difference is in the spelling. Cases like this can probably be set aside as marginal, leading to the view that, to a first approximation, the words of the language do indeed form a digital system.

To what extent do these considerations lend support to the claim that letters, words, and sentences are generically dependent entities? From the preceding discussion, it seems clear that a set of elements forming a digital system are thereby endowed with a robust criterion of identity, in the sense that any token of one of these elements is unequivocally a token of that element, sharply distinct from any other element - and this is true notwithstanding the possibility that in practice it may be difficult to determine which element it is a token of or even whether it is a token all. ${ }^{18}$ This is clearest for letters,

\footnotetext{
${ }^{18}$ To illustrate this latter case, suppose that when walking through the forest you come across three sticks on the ground so
} 
and somewhat less clear for words; for sentences, as syntactic entities, if it is assumed that two sentencetokens are tokens of the same sentence if and only if they are concatenations of tokens of exactly the same words, in the same order, then the case for sentences is no less clear than the case for words. In the same way, this could be applied to extended sequences of sentences - texts - so long as all that matters is their syntactic identity, in which case there is no essential difference between a whole text and a single sentence. In each case, there is a concatenation of words, and the identity of the whole is a function of the identities of the component words.

With propositional content, things become considerably more complicated. Here what is at issue is the meanings of sentences, and not just the sequences of words of which they are composed. Propositional content types do not form a digital system in Haugeland's sense, since they do not constitute a 'prespecified' set - at least not unless a thoroughgoing form of logical atomism is true. Whereas letters and words have a discrete 'all or nothing' character about them, this does not seem to be the case with propositions. One proposition can mean very nearly, but not quite, the same thing as another. Something like this happens with translation: to translate a text completely faithfully from one language to another would be to replace a token which bears a certain propositional content $P$ by virtue of its verbal content $V$ by a token which bears the same propositional content by virtue of bearing a verbal content $V^{\prime}$ which is completely different from $V$ since its constituent words are in a different language. ${ }^{19}$ In reality the most one can usually hope for is a token with propositional content $P^{\prime}$ approximately the same as $P$ where what is meant by 'approximately the same' is itself hard to quantify. ${ }^{20}$

This kind of indeterminacy similarly infects texts when considered from the point of view of propositional content. Texts are not valued merely for their syntactic identity: a text may constitute a work, e.g., a novel, a poem or a playscript, and what matters about works is not just their syntactic identity but what may be called their "semantic identity", an identity which is a function of, amongst other things, the propositional content of its constituent sentences. Unlike syntactic identity, semantic identity may be preserved under minor editorial amendments, under paraphrase, and, arguably, even under translation into a different language - so that, for example, a person swearing on an English bible may reasonably be considered to be swearing on the same literary work as her German counterpart. But this thought, if pursued to its logical conclusion, spells the end for any coherent criterion of identity for literary works. The point has been made forcefully by Goodman [14] in connection with musical works. If musical works are to have to have clear-cut identity criteria then, as Goodman points out, we have to insist that performances are note-perfect: a performance that purported to be of the Moonlight Sonata, but which contained a single wrong note (that is, a note differing from the note prescribed by Beethoven's score), would, for Goodman, not be a performance of that sonata but of a very similar work which differs from it in that one note. As Goodman himself concedes, this rather exacting standard of musical identity is completely at variance with commonly accepted ideas of musical performance. ${ }^{21}$

Thus although literary and musical works are widely considered to be entities generically dependent on their tokens, this becomes problematic if the entities populating an ontology are required to have robust identity criteria. But because literary works are not the same as texts, none of this impinges on the earlier conclusion that texts may be regarded as bona fide generically dependent entities. I cannot here pursue the many interesting additional ontological questions raised by artworks.

configured as to resemble the letter ' $\mathrm{A}$ '. Whether or not this actually is a token of ' $\mathrm{A}$ ' depends on whether or not the sticks were assembled in that configuration by someone with the intention of tokening the letter 'A', and it may be impossible for you to determine whether or not this is the case.

${ }^{19}$ Here there is an implicit assumption that words in different languages are necessarily distinct units of verbal content, but in fact we often say things like 'We have the same word in English'. For our present purposes, I'm not sure that much hangs on this.

${ }^{20}$ Compare this with transliteration, in which a token which bears a certain verbal content $V$ by virtue of bearing graphemic content $G$ is replaced by a token bearing the very same verbal content by virtue of bearing a different graphemic content $G^{\prime}-$ and here there is no need to compromise on 'very same' since $100 \%$ transliteration fidelity is undoubtedly possible.

${ }^{21}$ For further discussion of this and related points, see Simons $[30, \S 7.6]$. 


\section{Case Study III: Collectives}

Collectives of various kinds play a prominent role in our descriptions of the world, and have in recent years become the focus of increasing attention from ontologists within both philosophy and computer science $[30,38,11,26]$. In [38] a distinction is made between collectives whose membership is constant, so that, at all times at which the collective exists at all, its members are the same, and collectives with variable membership. Amongst constant-membership collectives a further distinction can be made between those whose membership is necessarily constant because having those particular members is part of the definition of the collective, and those whose membership is contingent, meaning that members could leave or be replaced without compromising the identity of the collective, although as chance would have it, this never occurs. The significant distinction here is surely that between collectives whose membership is necessarily constant, obeying the rule

$$
\square \forall x \forall t \forall t^{\prime}\left(\operatorname{Member}(x, c, t) \leftrightarrow \operatorname{Member}\left(x, c, t^{\prime}\right)\right)
$$

(where $c$ is the collective) and those whose membership can vary (whether or not it actually does so), obeying the rule

$$
\diamond \exists x \exists t \exists t^{\prime}\left(\operatorname{Member}(x, c, t) \wedge \neg \operatorname{Member}\left(x, c, t^{\prime}\right)\right)
$$

which is, of course, equivalent to the negation of (CM). Simons [30, $§ 4.4]$ calls the former kind 'classes' and the latter 'groups', and notes that classes are a 'limiting case' of groups. In this paper I shall reserve the term 'collective' for the variable-membership case (Simons' groups), and use 'collection' for the constant-membership case (Simons' classes).

A collection, in this sense, is specifically dependent on its members. It exists only so long as those members all exist. The surest way of naming a collection is simply by listing its members, for example Io, Europa, Ganymede and Callisto. This particular collection is also referred to as the Galilean moons, meaning that they are the four moons which Galileo discovered orbiting round Jupiter; but they are also the four largest moons of Jupiter, so another way of referring to them is to use the phrase just italicised. This phrase, however, unlike 'the Galilean moons', or the listing of names, is susceptible to two distinct interpretations. Under the de re reading, it refers again to that collection of four bodies which are currently Jupiter's four largest moons, that is, the Galilean moons. This is the sense that must be intended if one says that, as a result of some cosmic cataclysm, the four largest moons of Jupiter will, in five million years' time, be in orbit round Saturn. Under the de dicto reading, on the other hand, the same phrase refers to a collective, whose members at any one time are those bodies which happen to be the four largest moons of Jupiter at that time, and this is the sense that would be intended if one were to say that, as a result of the same cosmic cataclysm, the four largest moons of Jupiter will be Amalthea, Himalia, Thebe, and Elara.

Collectives are generically dependent on types: in this case, moons, or, more specifically, moons of Jupiter. If $c_{\text {FLMJ }}$ is the collective picked out by the de dicto reading of the italicised phrase, then we have

$$
\square\left(\operatorname{Exists}\left(c_{\mathrm{FLMJ}}\right) \rightarrow \exists x M \text { oonOf }(x, \text { jupiter })\right)
$$

which by (GD) means that $c_{\mathrm{FLMJ}}$ is generically dependent on the type 'moon of Jupiter'. This is a case of conjoint multiple generic dependence, but as in $\S 2$ it entails also a singular generic dependence of $c_{\text {FLMJ }}$ on elements of type 'collection of four moons of Jupiter', each of which is specifically dependent on elements of the type 'moon of Jupiter'. Thus there is a tight set of interrelationships represented diagrammatically in Figure 2, where $F$ can be read for the purposes of this example as 'moon of Jupiter'. In this diagram, generic dependences are shown linking individuals to types, specific dependence linking individuals to individuals, and instantiation linking types to individuals, in accordance with the ontological nature of these relationships. 


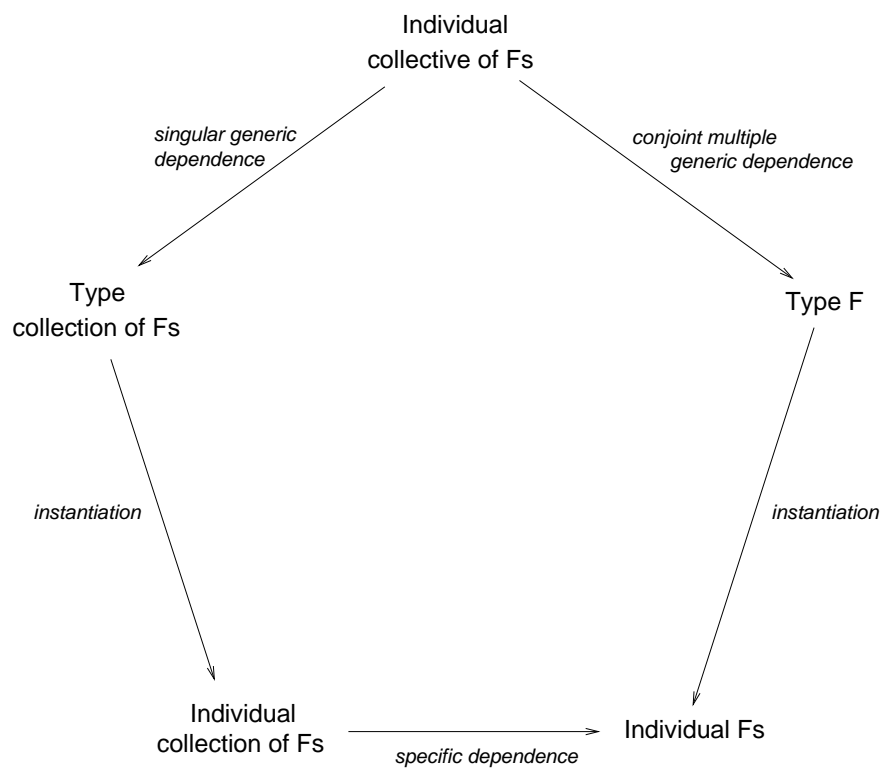

Figure 2: Dependence for collectives and collections

If collectivity is really a case of generic dependence it should not be possible for a collective to exist without any members. In [38] it is noted that some authors require a collective to have at least two members in order to exist, some require at least one, but yet others allow a collective to continue to exist even at times when it has no members. Thus in [26], 'Empty collectives are allowed. This is convenient when we want to talk about concentrations of zero or things that are empty or missing'. This approach was rejected in [38] on the grounds that it is 'contrary to the spirit of treating a collective as a concrete particular', and if this point of view is taken then it is, so far, consistent to regard collectives as generically dependent on their membership.

Turning to questions of identity for collectives, note that these appear in rather different guises depending on whether collectives are considered as having singular generic dependence on the collections that constitute them, or conjoint multiple generic dependence on their members. In the former case, since a collective can be constituted by only one collection at a time, the key question of identity is the diachronic one concerning whether collections existing at different times constitute the same collective:

- Is the collective constituted by collection $c_{1}$ at time $t_{1}$ the same collective as the collective constituted by collection $c_{2}$ at $t_{2}$ ?

In the latter case, the question takes the following form:

- Does individual $x_{1}$ belong to the same collective at time $t_{1}$ as individual $x_{2}$ at time $t_{2}$ ?

This question has diachronic and synchronic variants, obtained by putting $x_{1}=x_{2}$ and $t_{1}=t_{2}$ respectively:

- (Diachronic:) Does individual $x$ belong to the same collective at times $t_{1}$ and $t_{2}$ ?

- (Synchronic:) Do individuals $x_{1}$ and $x_{2}$ belong to the same collective at time $t$ ?

As stated, these questions may be too general to be answerable. Since an individual may belong to many different collectives at the same time, it is necessary to be more specific about what type of collective is being referred to: instead of 'Do they belong to the same collective?', the appropriate 
question is 'Do they belong to the same collective of type $C$ ?', for some collective type $C$ - for example swimming club, orchestra, queue, or species.

Even in this case, the questions may be ambiguous. Some types of collective are such that an individual cannot belong to more than one collective of that type at the same time, whereas for others no such restriction holds. I shall call the former type exclusive and the latter non-exclusive. At a given time, a person can belong to more than one choir, more than one orchestra, more than one club, and so on, so these are non-exclusive collectives; but a student cannot belong to more than one year-group, a tree cannot belong to more than one forest, and a living organism cannot belong to more than one species, and these are accordingly exclusive collectives. For non-exclusive collectives, the question 'Do $x_{1}$ and $x_{2}$ belong to the same $C$ ?' can be interpreted either as 'Is there any $C$ that $x_{1}$ and $x_{2}$ both belong to?' or as 'Do they belong to all the same $C$ s?'. For these collectives, identity questions are best handled at the level of their constituent collections rather than their individual members. For exclusive collectives, on the other hand, one can meaningfully refer to the collective of type $C$ to which a given individual belongs, so long as that individual belongs to some collective of that type. As always, the crucial question concerns the transitivity or otherwise of the relation 'belongs to the same $C$ as'. Failure of transitivity would render the identity of $C$ s problematic.

The case of species is particularly interesting. Biologists have had considerable difficulty formulating a satisfactory definition of species. The problem is that unless the relation used to define conspecificity (that is, belonging to the same species) is transitive, it will not be possible to partition the set of all organisms into equivalence classes that can play the role of species. A widely-used definition of species is that individuals are conspecific if they belong to populations which can interbreed to produce fertile offspring, but this is well-known not to be transitive, giving rise to sorites series of populations in which neighbouring pairs are conspecific but the end-points are not (as famously illustrated by the Herring Gull / Lesser Black-backed Gull complex [24]). Despite the existence of such problematic cases, it seems that at a given time, most individuals belong clearly to one species or another, but when individuals separated in time are considered, the indeterminacy of species boundaries kicks in with a vengeance, as pointed out persuasively by Dawkins [7, pp.258f].

There seem to be two different ways of looking at this, which I call the three-dimensional view, and the four-dimensional view. ${ }^{22}$ On the three-dimensional view, a species is a continuant. It comes into existence at a certain point in geological time, endures for some period, and then ceases to exist, either by becoming extinct, or by evolving into one or more successor species. At any time of its existence, the species is present as a whole: it is, precisely, a variable-membership collective, and in the course of its history, it undergoes a continuous replacement of members. Viewed in this way, at any one time the species is constituted by some maximal collection of conspecific individuals. A problem of species identity arises in any case where it is not possible to determine conspecificity. As noted above, for many species, during the greater part of their period of existence, the members of the species are clearly distinct from the members of any other species. For example, there is presumably no individual now alive of which it is uncertain whether or not it is a lion. This cannot always have been the case, however, since looking back far enough in the evolutionary history of this species, one will find a time when it is not possible to distinguish sharply between the collective of lion-ancestors and the collective of tiger-ancestors - this is essentially the point made by Dawkins which I cited earlier. If the species is considered over just the portion of its evolutionary history when it is clearly distinct from all other species - and given that speciation appears to be a relatively rapid process, when viewed from the evolutionary time-scale, it is likely that for many species, this portion will amount to the greater part of their history — then during this period of time the species can reasonably be viewed as a well-defined entity that is generically dependent on its members.

The four-dimensional view of species is rather different. On this view, a species is not a continuant

\footnotetext{
${ }^{22}$ These two views correspond, at least approximately, to the distinction drawn in [34] between species as collections and species as classes.
} 
but something more like (but not exactly) an occurrent, which comprises the members from throughout its history, and thus is not a collective in the sense in which this term is understood here. The species Homo sapiens, thus considered, is the aggregate of all humans, past, present, and future. Whereas on the three-dimensional view the membership of the species is constantly changing over time, on the fourdimensional view it has a fixed membership but at any given time only some of those members are present. Although a four-dimensional collective, thus conceived, satisfies the formula (CM) above, this does not make its dependence on its members rigid, since although each of its members is a member of it timelessly, its membership is not in every case necessary - for example, there could have been humans different from those that actually have been, are, or will be.

This contrast between three- and four-dimensional views of species could in principle be extended to other ensembles which are normally thought of as collective continuants. In many such cases, the four-dimensional view seems much less natural than the three-dimensional. A choir of any longevity, for example the Leeds Festival Chorus, will have a wholesale turnover of its members during the course of its existence. One could view it as a four-dimensional aggregate whose members are all the people who have been, are, or will be members of it in the three-dimensional sense. This would imply that many members of the chorus are dead, and some have yet to be born. It is not surprising that most of the time we find it more convenient to deal with collectives as continuants. As such, their generic dependence on their membership is of the conjoint multiple kind, and is determinate to the extent that there are clear membership criteria: reasonably firmly so in the case of choirs, typically less so in the case of species.

\section{Conclusion}

In this paper I have considered the phenomenon of generic dependence both in general terms and in relation to a series of concrete case studies. I have placed considerable emphasis on questions of identity for generically dependent entities, and the case studies have shown that if identity is considered important then the ontological status of some alleged generically dependent entities may be cast in doubt.

In the case of shapes, for example, because the notion of sameness of shape has to be relativised to the resolution of some measurement procedure, there can be no absolute criterion of identity for the shapes of physical objects, so long as these shapes are considered to be generically dependent entities capable of multiple realisation, casting doubt on the viability of this notion. And if instead, the shape is regarded as a specifically dependent entity inalienably tied to a unique individual, then the same problem infects the qualia which provide its possible values.

Syntactic elements such as letters, words, and sentences seemed to offer us a more clear-cut case where the notion of generic dependence could gain purchase, for here, since these kinds of elements form digital systems in the sense of Haugeland [15], it does appear that they possess sufficiently robust criteria of identity to sustain their existence as entities - albeit dependent ones - within an ontology. The dangers of extrapolating this to more "semantic" entities such as literary works were alluded to, but not explored in depth.

The final case study concerned collections and collectives. A collection is defined extensionally and as such has constant membership; its dependence on its members is specific, not generic. This specific dependence of a collection on its members is analogous, not to the generic dependence of a text on its tokens, but rather to the specific dependence of a text on its constituent words. Both collection/members and text/words are instances of a mereologically constant entity being specifically dependent on its parts. ${ }^{23}$ Thus generic dependence only arises as an issue in relation to collectives, not collections. And in the case of collectives, at least in some cases, non-robust identity arises from the possibility of vicious sorites series. I illustrated this in the case of biological species, but there are plenty of other examples.

\footnotetext{
${ }^{23}$ Note, however, that the contrary view has also been advanced, that the parts are dependent on the whole, on the grounds that the parts cannot exist as parts unless the whole exists. For a discussion of this see, for example, [17, pp.112ff].
} 
To cite just one other, consider the notion of a generation. One hears about 'the older generation', 'the younger generation', 'people of my generation', or 'the generation that grew up in the sixties'. People even talk about 'the next generation', as if there were a discrete sequence of clearly separate generations succeeding one another in time. A generation would appear to be a kind of collective, yet it obviously suffers from vicious problems of identity, which become apparent if you consider a sequence of individuals born in each successive year from 1950 to 2000, clearly illustrating the non-transitivity of the same generation relation, and therefore the non-robustness of generational identity.

Have we become too fixated on questions of identity? Concepts such as 'species', 'shape', 'artwork', and 'generation' are to a considerable extent comfortably enshrined in everyday discourse, their ontologically questionable character being somehow glossed over much of the time. Doubts about the identity of generically dependent entities arise from the possibility, in principle, of sorites series exhibiting smooth transitions between distinct instances of their category. ${ }^{24}$ But one might argue that such sorites series are seldom, if ever, met with in practice. It may well be accepted that, ultimately, these generically dependent entities are susceptible to sorites-based identity crises, but because this possibility is seldom encountered in reality except when specifically engineered for the purposes of exhibiting precisely such a possibility, it is useful to resort to the convenience of speaking and thinking about them without worrying about their true ontological status.

What, then, is their "true ontological status"? In calling any entity dependent, one is already relegating it to second-class citizenship of the ontological realm. But opinions differ as to whether such relegation is tantamount to banishing such entities altogether. If the entity $X$ is generically dependent on entities of type $Y$, does this mean that $X$ does not "really" exist, only the $Y \mathrm{~s}$, or does it rather vindicate the claim that $X$ exists by grounding it in entities whose existence is in some way more fundamental or less problematic? Here it is useful to distinguish three possible stances, which in the terminology of Baker [2] are called non-reductionism, reductionism, and eliminativism. The non-reductionist takes the view that even when $X$ is dependent on the $Y \mathrm{~s}, X$ cannot be explained away through this dependence: $X$ exists over and above the $Y$ s on which it ontologically depends. Note that this is uncontroversial in the case of historical dependence [35] — I certainly exist over and above my parents, even though I am historically dependent on them. But it is more contentious for constant dependence. The reductionist will still accept that the dependent entity exists, but its existence amounts to "nothing but" the existence of the entities on which it depends. On this view, $X$ exists by virtue of the $Y$ s; everything said about $X$ could in principle be cashed out in terms of things that can be said about the $Y \mathrm{~s}$, but the virtual impossibility of doing this in practice makes it convenient to continue talking about $X$. Eliminativists takes a more uncompromising stance: for them, $X$ simply does not exist. The only things that exist are the "fundamental" entities which are not dependent on anything else.

It is not always easy to tell whether someone speaking in a broadly reductionist spirit is a reductionist

\footnotetext{
${ }^{24}$ This situation should not be confused with the case of broad categories characterised in terms of resemblance to a prototype. To illustrate the difference, consider the case of colour. We distinguish between, on the one hand, broad chromatic categories corresponding to colour-words such as 'red', 'green', 'indigo', and 'violet', and on the other hand, the full range of discriminable shades exhibited by individual coloured samples. We use the term 'colour' for the former, and 'hue' for the latter. Colour is a prototypical exemplar of a category system organised in terms of prototypical exemplars. As Berlin and Kay say in their pioneering study of basic colour terms, "[r]epeated mapping trials with the same informant and also across informants showed that category foci placements are highly reliable. ... Category boundaries, however, are not reliable, even for repeated trials with the same informant' $[3, \S 1.6]$. Prototype theory is unembarrassed by sorites colour series precisely because such series must cross these ill-defined category boundaries whose significance to the theory is minimal. Contrast the case of colour with that of hue. Ideally, the space of possible hues is continuous, with each hue specified in terms of real-valued parameters, for example an RGB triple. But if we wish to reify hues as entities generically dependent on individual physical colour samples, we encounter the same situation as with shapes, in which we can only discriminate up to a certain level of resolution determined by our measuring instruments. A generically dependent hue should be dependent on all and any of a collection of samples having the same hue; but in reality we must make do with discriminable hue, and as with shape, indiscriminability is not an equivalence relation. Category boundaries are not an issue here; the identity of individual hues is already problematic before we start collecting hues together into broad colour categories.
} 
or an eliminativist. A famous case is the notorious remark by the former British prime minister Margaret Thatcher that 'there is no such thing as society', ${ }^{25}$ a remark for which she continues to be widely reviled in left-wing circles. What she actually said was 'And, you know, there is no such thing as society. There are individual men and women, and there are families'. Taken literally, her words can be understood as expressing an eliminativist stance towards society: literally, society does not exist, all there is is individual men and women and families. (She might as well have gone on to say that families do not exist either, just men, women, and children.) Or she could be understood as expressing a reductionist view, that although society does exist, its existence amounts to nothing over and above the individuals who compose it (together with, of course, all the myriad ways in which they interact - though Thatcher was silent about these). The political ramifications of these two distinct interpretations lie outside the scope of this paper, but I believe they are considerable, and much of the subsequent commentary on Thatcher's remark can be seen as an attempt to clarify them. What seems undeniable, however, is that she was trying to repudiate the non-reductive view of society, according to which society acquires some sort of emergent identity over and above that of the individuals and their activities on which it depends - a view which she would have associated, rightly or wrongly, with the socialism which she was so antagonistic to.

In conclusion, I would like to propose that although ontology is, ostensibly, the study of what exists, the notion of "existence" is not really what is primarily of interest. The real significance of the kinds of argumentation in this and kindred papers may be that the primary business of metaphysics and ontology should be not so much the question of "what exists" — important though that may be — as the tracing of the intricate network of dependencies amongst the rich array of different kinds of entity that we speak about, for it is ultimately this network of dependencies that defines the structure of our world as we see it and describe it.

\section{References}

[1] John Bacon. Tropes. In Edward N. Zalta, editor, The Stanford Encyclopedia of Philosophy. Winter 2011 edition, 2011.

[2] Lynne Rudder Baker. The Metaphysics of Everyday Life: An essay in practical realism. Cambridge University Press, Cambridge, UK, 2007.

[3] Brent Berlin and Paul Kay. Basic Color Terms: Their Universality and Evolution. University of California Press, Berkeley and Los Angeles, 1969.

[4] Fabrice Correia. Existential Dependence and Cognate Notions. Philosophia Verlag, Munich, 2005.

[5] Fabrice Correia. Ontological dependence. Philosophy Compass, 35:1013-32, 2008.

[6] L. da Fontoura Costa and R. M. Cesar. Shape Analysis and Classification: Theory and Practice. CRC Press, 2001.

[7] Richard Dawkins. The Ancestor's Tale. Weidenfeld and Nicolson, London, 2004.

[8] Kit Fine. Ontological dependence. Proceedings of the Aristotelian Society, 95:269-89, 1995.

[9] Luciano Floridi. The Philosophy of Information. Oxford University Press, Oxford, 2011.

[10] Gottlob Frege. Die Grundlagen der Arithmetik. Wilhelm Koebner, Breslau, 1884. English translation The Foundations of Arithmetic by J. L. Austin, Oxford: Basil Blackwell, 1950.

\footnotetext{
${ }^{25}$ Interview with Women's Own magazine, 31st October 1987. She had previously made a similar remark in her speech to the Conservative Party Conference on 9th October 1987.
} 
[11] Antony Galton. How is a collection related to the sum of its members? In Barry Smith and Riichiro Mizoguchi, editors, Proceedings of InterOntology 2010, Tokyo, Japan, 2010.

[12] Antony Galton. Prolegomena to an ontology of shape. In O. Kutz, M. Bhatt, S. Borgo, and P. Santos, editors, Proceedings of the Second Interdisciplinary Workshop "The Shape of Things", Rio de Janeiro, Brazil, April 3-4, 2013, 2013. http://ceur-ws.org/Vol-1007/invited4.pdf.

[13] Peter Gärdenfors. Conceptual Spaces: the geometry of thought. MIT Press, Cambridge MA and London UK, 2000.

[14] Nelson Goodman. The Languages of Art. Oxford University Press, 1969.

[15] John Haugeland. Artificial Intelligence: The Very Idea. MIT Press, Cambridge, Massachussetts, 1985.

[16] IFOMIS. Basic formal ontology (BFO). http://www.ifomis.org/bfo, Accessed 21/12/2012, 2009.

[17] Kathrin Koslicki. The Structure of Objects. Oxford University Press, 2008.

[18] Kathrin Koslicki. Varieties of ontological dependence. In Fabrice Correia and Benjamin Schnieder, editors, Metaphysical Grounding, pages 186-213. Cambridge University Press, 2012.

[19] Kathrin Koslicki. Ontological dependence: An opinionated survey. In Benjamin Schnieder, Miguel Hoeltje, and Alexander Steinberg, editors, Dependence, Emergence, Supervenience. Philosophia Verlag, München, forthcoming.

[20] E. Jonathan Lowe. The Four Category Ontology. A Metaphysical Foundation for Natural Science. Oxford University Press, Oxford, 2006.

[21] E. Jonathan Lowe. Ontological dependence. In Edward N. Zalta, editor, The Stanford Encyclopedia of Philosophy. Spring 2010 edition, 2010.

[22] André Martinet. Eléments de Linguistique Generale. Colin, Paris, 1960.

[23] C. Masolo, S. Borgo, A. Gangemi, N. Guarino, and A. Oltramari. WonderWeb deliverable D18. Technical report, Laboratory for Applied Ontology, ISTC-CNR, Trento, Italy, 2003.

[24] Ernst Mayr. Systematics and the Origin of Species. Columbia University Press, New York, 1942.

[25] Florian Probst. Observations, measurements and semantic reference spaces. Applied Ontology, 3:63-89, 2008.

[26] A. Rector, J. Roger, and T. Bittner. Granularity, scale and collectivity: When size does and doesn't matter. Journal of Biomedical Informatics, 39(3):333-349, 2006.

[27] Robert Rovetto. The shape of shapes: An ontological exploration. In J. Hastings, O. Kutz, M. Bhatt, and S. Borgo, editors, SHAPES 1.0: The Shape of Things 2011 (Proceedings of the First Interdisciplinary Workshop), volume 812. CEUR Workshop Proceedings, 2011.

[28] Luc Schneider. The ontological square and its logic. In C. Eschenbach and M. Grüninger, editors, Formal Ontology in Information Systems: Proceedings of the Fifth International Conference (FOIS 2008), pages 36-48. IOS Press, 2008.

[29] Claude E. Shannon and Warren Weaver. The Mathematical Theory of Communication. University of Illinois Press, Urbana, 1949. 
[30] Peter Simons. Parts: a Study in Ontology. Clarendon Press, Oxford, 1987.

[31] Aaron Sloman. What's information, for an organism or intelligent machine? How can a machine or organism mean? http://www.cs.bham.ac.uk/axs/misc/whats-information.pdf, 2010.

[32] Barry Smith. Basic concepts of formal ontology. In Nicola Guarino, editor, Formal Ontology in Information Systems: Proceedings of FOIS'98, Trento, Italy, 6-8 June 1998, pages 19-28. IOS Press, Amsterdam, 1998.

[33] Barry Smith. Basic formal ontology. http://icbo.buffalo.edu/Presentations/Smith.ppt, 2009.

[34] Barry Smith. Biometaphysics. In Andrew McGonigal Robin Le Poidevin, Peter Simons and Ross P. Cameron, editors, The Routledge Companion to Metaphysics, pages 537-544. Routledge, Abingdon, 2009.

[35] Amie Thomasson. Fiction and Metaphysics. Cambridge University Press, 1999.

[36] Linda Wetzel. Types and tokens. In Edward N. Zalta, editor, The Stanford Encyclopedia of Philosophy. Spring 2011 edition, 2011.

[37] Ludwig Wittgenstein. Philosophical Investigations. Basil Blackwell, Oxford, 1958. English translation by G. E. M. Anscombe.

[38] Zena Wood and Antony Galton. A taxonomy of collective phenomena. Applied Ontology, 4:267$292,2009$. 